\title{
ADDERS FOR THE PATTERNED STARTER IN NONABELIAN GROUPS
}

\author{
KENNETH B. GROSS and PHILIP A. LEONARD \\ (Received 10 September 1974; revised 3 February 1975)
}

\begin{abstract}
Starters with adders, in abelian groups of odd order, have been used widely in recent combinatorial constructions, most notably in the study of Room squares. In this paper, constructions of adders for the so-called "patterned starter" are described, for a large class of nonabelian groups of odd order.
\end{abstract}

\section{Introduction}

Among the many algebraic tools which have been used in combinatorial constructions are starters, and starters with adders, in finite abelian groups of odd order. The concept of a starter with an adder in an abelian group appeared originally as a method in the construction of Room designs, see Mullin and Nemeth $(1969 \mathrm{a}, \mathrm{b})$. Later this technique was seen to be valuable in the study of one-factorization of complete graphs, Wallis (1973), Gross (1973), Anderson (1974). Recently, the concepts of a starter and an adder have been valuable tools in the study of Steiner systems, Gross (1973, 1974), neofields, Gross (1973), Howell designs, Stephen, Hung and Mendelsohn (1974), and mutually complementary topologies on finite sets, Anderson (1973). It would seem that more applications will soon be found.

The problems arising in these various areas can often be dealt with by using adders for a class of distinguished starters called the patterned starters for abelian groups. The problem of the existence of adders for patterned starters in abelian groups has been solved for most abelian groups, Gross and Leonard.

\section{Patterned Starter}

If $G$ is any finite group of odd order, we let $G^{*}$ denote $G \backslash\{1\}$, where 1 denotes the identity of $G$. A starter $X$ for $G$ is a partition of $G^{*}$ into 2-sets such that

$$
\left\{x y^{-1} \mid\{x, y\} \in X\right\}=G^{*} .
$$


If $G$ is as above and $X$ is a starter for $G$, then a left-handed adder for $X$ is an injection $A: X \rightarrow G^{*}$ with the property that

$$
\bigcup_{\{x, y\} \in X}\{\{x, y\} A x,\{x, y\} A y\}=G^{*} \text {. }
$$

Similarly, a right-handed adder for $X$ is an injection $A: X \rightarrow G^{*}$ with the property that

$$
\bigcup_{\{x, y\} \in X}\{x\{x, y\} A, y\{x, y\} A\}=G^{*} .
$$

We point out that if $G$ is abelian, then every left-handed adder for $X$ is a right-handed adder for $X$, and vice versa. In this case, and in others, where no confusion will arise, we use the term "adder" alone to describe the map $A$.

Given a starter $X$ for a group $G$ and a left-handed adder (right-handed adder) for $X$, a Room design $R$ of side $|G|$ can be constructed as follows: let $R$ be a square array with rows and columns indexed by the elements of $G$. The cell $(g, h)$ (in row $g$, column $h$ ) is left empty unless either (i) $g^{-1} h=$ $(\{x, y\} A)^{-1}\left(h g^{-1}=(\{x, y\} A)^{-1}\right)$ for some $\{x, y\} \in X$, or (ii) $g^{-1} h=1$. In case (i), we place the pair $\{g x, g y\}(\{x g, y g\})$ in cell $(g, h)$. In case (ii), we place the pair $\{\infty, g\}$ in cell $(g, h)$, where $\infty$ is a distinguished element not contained in $G$.

For a more detailed discussion of this procedure in the case of abelian groups, as well as for background material on Room designs, the reader is referred to the work of Mullin and Nemeth (1969b) and Wallis, Street and Wallis (1972).

Each group $G$ of odd order possesses a distinguished starter $P S(G)$ known as the patterned starter for $G$, referred to as the patterned starter when there will be no confusion about the group, defined by

$$
P S(G)=\{\{x, y\} \mid x y=1, \text { and } x \neq 1\} .
$$

If $G$ is an abelian group, the existence of an adder for $P S(G)$ is equivalent to the existence of a strong starter for $G$, Byleen (1970) (a starter $X$ for $G$ with the property that $\left.\{x y \mid\{x, y\} \in X\}=G^{*}\right)$. The existence question for adders for $P S(G)$ has been settled affirmatively for abelian groups $G$ whose orders are not divisible by 3 or 5, and for many other cases, Mullin and Nemeth (1969) and Gross and Leonard (to appear).

The question of the existence of adders for starters in non-abelian groups seems to have been first raised in Wallis, Street and Wallis (1972). Since then Leonard (1974), examples have been found of right-handed adders for the patterned starter in the non-abelian groups of order $p q$, where $q \equiv p \equiv 3(\bmod$ 4) and $q \equiv 1(\bmod p)$. In this paper we consider the problem from the standpoint of group extensions, and show how to construct adders for $P S(G)$ in a much wider class of non-abelian groups. 


\section{Computations}

Since patterned starters only exist for groups of odd order, and all groups of odd order are known to be solvable, Feit and Thompson (1963), it is sufficient to construct adders for the patterned starter in solvable groups. Our main results will rely on an inductive argument based on the theory of group extensions. In particular, if $G$ is solvable, then $G$ has a normal subgroup $H$. Let $\Phi: G / H \rightarrow K$ be an isomorphism of $G / H$ onto a group $K$. Let $X=\left\{x_{k} \mid k \in K\right\}$ be a left-transversal of $H$ in $G$, where $x_{k} \Phi=k$ and $x_{1}=1$. The elements of $X$ act on $H$ by conjugation, and we write $h^{k}=x_{k}^{-1} \cdot h \cdot x_{k}$ to indicate the action of $x_{k}$ on $h$.

Multiplication in $G$ can be expressed in terms of that for $H$ and $K$ :

$$
x_{k} h \cdot x_{k} \cdot h^{\prime}=x_{k k^{\prime}} \cdot m_{\left(k, k^{\prime}\right)} \cdot h^{k^{\prime}} \cdot h^{\prime}
$$

where $m_{\left(k, k^{\prime}\right)}$ is an element of $H$ such that $x_{k} \cdot x_{k^{\prime}}=x_{k k^{\prime}} \cdot m_{\left(k, k^{\prime}\right)}$. We will refer to the system of factors $\left\{m_{\left(k, k^{\prime}\right)}\right\}$ as a factor system. Two factor systems $\left\{m_{(a, b)}\right\}$ and $\left\{n_{(a, b)}\right\}$ will be said to be distinct if there exist $k, k^{\prime} \in K$ such that $m_{\left(k, k^{\prime}\right)} \neq n_{\left(k, k^{\prime}\right)}$. Inverses are given by $\left.\left(x_{k} h\right)^{-1}=x_{k^{-1}} \cdot\left(h^{k^{-1}}\right)^{-1} \cdot m_{(k, k)}^{-1}\right)$.

Now, let $T$ denote a maximal set of elements of $K$ containing no inverses. Given $P S(H)$ and $P S(K)=\left\{\left\{c, c^{-1}\right\} \mid c \in T\right\}$, it is possible to express $P S\left(G^{\prime}\right.$ in the form

$$
\left.P S(G)=P S(H) \cup\left\{\left(x_{c} h\right),\left(x_{c} h\right)^{-1}\right\} \mid c \in T, h \in H\right\} .
$$

With these notations in mind, we have the following

Theorem 1. If $A_{H}$ and $A_{K}$ are left-handed adders for PS $(H)$ and $P S(K)$, respectively, and if for each $c \varepsilon T$ there is a permutation $\pi_{c}$ of $H$ satisfying

(i) $h \rightarrow\left(h \pi_{c}\right)^{c} \cdot h$ is a permutation of $H$, and

(ii) $h \rightarrow\left(h \pi_{\mathrm{c}}\right) \cdot h^{-1}$ is a permutation of $H$, then the map $A: P S(G) \rightarrow G^{*}$ defined by the following rules defines a lefthanded adder for $P S(G)$ :

$$
\left\{h, h^{-1}\right\} A=\left\{h, h^{-1}\right\} A_{H},
$$

$\left\{x_{c} h,\left(x_{c} h\right)^{-1}\right\} A=x_{c A_{K}} \cdot h \pi_{c} \quad$ for $c \in T, h \in H$, where "c $c A_{K} "$ denotes $\left\{c, c^{-1}\right\} A_{K}$.

PROOF. It suffices to check the products of $x_{c A_{K}} \cdot h \pi_{c}$ with $x_{c} h$ and $\left(x_{c} h\right)^{-1}$. We have

$$
x_{c A_{K}} \cdot h \pi_{c} \cdot x_{c} \cdot h=x_{c A_{K}, c} \cdot m_{\left(c A_{K}, c\right)} \cdot\left(h \pi_{c}\right)^{c} \cdot h,
$$

and

$$
\begin{gathered}
x_{\mathrm{cA} K} \cdot h \pi_{\mathrm{c}} \cdot\left(x_{c} h\right)^{-1}=x_{\mathrm{CA}, c^{-1}} \cdot m_{\left(c A_{K, c} c^{-1}\right)} \cdot\left(h \pi_{c}\right)^{c^{-1}} \cdot\left(h^{c^{-1}}\right)^{-1} \cdot m_{\left(c, c^{-1}\right)}^{-1} \\
=x_{c A_{K} \cdot c^{-1}} \cdot m_{\left(c A_{K}, c^{-1}\right)} \cdot\left(h \pi_{c} h^{-1}\right)^{c^{-1}} \cdot m_{\left(c, c^{-1}\right)}^{-1} .
\end{gathered}
$$


Since $A_{K}$ is an adder for $P S(K)$, the elements $x_{c A_{K} c}$ and $x_{c A_{K} c^{-1}}$ for $c \in T$ make up all of the elements $x_{k}, k \in K$, except for $k=1$. For fixed $c$, as $h$ runs over the elements of $H$, so do the right-hand sides of (a) and (b). Thus the function defined by

$$
\begin{aligned}
& x_{c} \cdot h \rightarrow\left\{x_{\mathrm{c}} \cdot h,\left(x_{\mathrm{c}} \cdot h\right)^{-1}\right\} A \cdot x_{\mathrm{c}} \cdot h, \\
& \left(x_{c} \cdot h\right)^{-1} \rightarrow\left\{x_{c} \cdot h,\left(x_{c} \cdot h\right)^{-1}\right\} A \cdot\left(x_{c} \cdot h\right)^{-1}, \quad \text { for } c \overline{E T}
\end{aligned}
$$

is a permutation of $K^{*}$. It follows that property (2.1) holds. Thus, $A$ is an adder for $P S(G)$.

Looking over the hypotheses of Theorem 1, we note the following

COROLLARY. The answer to the question of whether $A$ is a left-handed adder for PS $(G)$ is independent of the factor system.

In order to illustrate the construction described in Theorem 1, we offer the following example. Let $Z_{7}$ denote the additive group of integers modulo 7 . Let $G=Z_{7} \oplus Z_{7}, K=Z_{7}$, and let $H$ be the subgroup of $G$ consisting of the elements $(0,0),(1,0),(2,0),(3,0),(4,0),(5,0),(6,0)$. Let $\{1,6\} A_{K}=2,\{2,5\} A_{K}=4$, and $\{3,4\} A_{K}=1$, and define $A_{H}$ to be the corresponding map on $P S(H)$. Let $T=\{1,2,3\}$. Define $h \pi_{\mathrm{c}}=2 h$. It is easily shown that $\pi_{\mathrm{c}}$ satisfies the conditions (i) and (ii) in Theorem 1 - we remind the reader that here we are working in an additive group. For each $c \in K$, we define $x_{c}=(0, c) \in G$.

We are thus able to write the elements of $P S(G)$ as in the following array, the first row being $P S(H)$ :

$\begin{array}{lll}\{(1,0),(6,0)\} & \{(2,0),(5,0)\} & \{(3,0),(4,0)\} \\ \{(0,1),(0,6)\} & \{(0,2),(0,5)\} & \{(0,3),(0,4)\} \\ \{(1,1),(6,6)\} & \{(1,2),(6,5)\} & \{(1,3),(6,4)\} \\ \{(2,1),(5,6)\} & \{(2,2),(5,5)\} & \{(2,3),(5,4)\} \\ \{(3,1),(4,6)\} & \{(3,2),(4,5)\} & \{(3,3),(4,4)\} \\ \{(4,1),(3,6)\} & \{(4,2),(3,5)\} & \{(4,3),(3,4)\} \\ \{(5,1),(2,6)\} & \{(5,2),(2,5)\} & \{(5,3),(2,4)\} \\ \{(6,1),(1,6)\} & \{(6,2),(1,5)\} & \{(6,3),(1,4)\}\end{array}$

And the map $A$ takes each of the pairs in the above array to the element in the corresponding position of the following array: 


$\begin{array}{lll}(2,0) & (4,0) & (1,0) \\ (0,2) & (0,4) & (0,1) \\ (2,2) & (2,4) & (2,1) \\ (4,2) & (4,4) & (4,1) \\ (6,2) & (6,4) & (6,1) \\ (1,2) & (1,4) & (1,1) \\ (3,2) & (3,4) & (3,1) \\ (5,2) & (5,4) & (5,1)\end{array}$

Theorem 1 tells us that $A$ is an adder for $P S(G)$.

In addition to Theorem 1, we have the following theorem for the case of right-handed adders:

TheOREM 2. If $A_{H}$ and $A_{K}$ are right-handed adders for $P S(H)$ and $P S(K)$, respectively, and if for each $c \in T$ there is a permutation $\pi_{c}$ of $H$ satisfying

(iii) $h \rightarrow h^{\mathrm{cA} A_{K}} \cdot h \pi_{\mathrm{c}}$ is a permutation of $H$, and

(iv) $h \rightarrow\left(h^{-1}\right)^{c^{-1} \cdot c A_{K}} \cdot m_{\left(c^{-1}, c A_{K}\right)} \cdot\left(m_{(c, c}^{-1}\right)^{c A_{K}} \cdot h \pi_{c}$

is a permutation of $H$, then the map $A$ defined as in Theorem 1 is a right-handed adder for $P S(G)$.

Proof. We note that

$$
x_{c} \cdot h \cdot x_{c A_{K}} \cdot h \pi_{c}=x_{c \cdot C A_{K}} \cdot m_{\left(c, c A_{K}\right)} \cdot h^{c A_{K}} \cdot h \pi_{c},
$$

and that

(b) $\left(x_{c} \cdot h\right)^{-1} \cdot x_{c A_{K}} \cdot h \pi_{c}=x_{c^{-1} \cdot c A_{K}} \cdot m_{\left(c^{-1}, c A_{K}\right)} \cdot\left(\left(h^{c-1}\right)^{-1} \cdot m_{\left(c, c^{-1}\right)}^{-1}\right)^{c A_{K}} \cdot h \pi_{c}$

$$
=x_{c^{-1} \cdot c A_{K}} \cdot\left(h^{-1}\right)^{c^{-1} \cdot c A_{K}} \cdot m_{\left(c^{-1}, c A_{K}\right)} \cdot\left(m_{\left(c, c^{-1}\right)}^{-1}\right)^{c A_{K}} \cdot h \pi_{c} .
$$

By an argument similar to the one given in Theorem 1, it becomes evident that $A$ is a right-handed adder for $P S(G)$.

It is not clear that the answer to the question of whether $A$ is a right-handed adder can always be considered to be independent of the factor system. When $\boldsymbol{H}$ is abelian, however, this is clearly true, since $m_{(a, b)} \in H$.

\section{Construction of adders}

When the problem of constructing adders for the patterned starter $P S(G)$ is viewed as in Theorems 1 and 2, it reduces to and

(a) finding appropriate adders for $P S(H)$ and $P S(K)$, 

ties.

(b) determining a set of permutations $\left\{\pi_{c} \mid c \in T\right\}$ with the required properIn several rather general situations, this can be accomplished rather easily. One example is given by

THEOREM 3. If $G=H \times K$, the direct product of $H$ and $K$, then if there are left- (right-) handed adders for PS $(H)$ and PS(K), then there is a left- (right-) handed adder for $P S(G)$ provided that $3 \nmid|H|$.

Proof. If this case, we may take $h \pi_{c}=h^{2}$ for all $c \in T$. Since all factors $\boldsymbol{m}_{(a, b)}$ are 1 , and all automorphisms performed by $x_{k}$ on $H$ are equal to the identity, in order to apply either Theorem 1 or Theorem 2 we need only show that

(i) $h \rightarrow h \pi_{c} \cdot h$ is a permutation of $H$, and

(ii) $h \rightarrow h \pi_{c} \cdot h^{-1}$ is a permutation of $H$.

As long as $|H|$ is not divisible by 3 , this is clearly true.

On the surface, Theorem 3 does not seem to be very helpful. However, we have immediately

Corollary 3.1. Let $G$ be a central extension of $H$ such that ||$H \mid$. Suppose that there is a left- (right-) handed adder for $P S(G / H)$ and an adder for $P S(H)$. Then there is a left- (right-) handed adder for $\operatorname{PS}(G)$.

Proof. Since $H$ is abelian, we may as well consider the direct sum $\bar{G}$ of $H$ with $G / H$, since only the factor system has changed. But, by Theorem $3, A$ is a left- (right-) handed adder for $P S(\bar{G})$. Thus, $A$ is a left- (right-) handed adder tor $P S(G)$.

COROLlary 3.1.1. If $G$ is a finite $p$-group with $p>5$ then PS(G) has both $a$ right-handed adder and a left-handed adder.

Proof. Every $p$-group can be considered a central expansion. We know from Gross and Leonard that there is an adder for the centre, and thus by induction an adder exists for every finite $p$-group.

Corollary 3.1.2. If $G$ is a finite nilpotent group such that $|G|$ is not divisible by 2,3 , or 5 , then there exists both a left-handed adder and a right-handed adder for $P S(G)$.

Proof. This follows from the fact that every finite nilpotent group can be decomposed into the direct product of $p$-groups.

We have not yet considered the type of construction introduced in Leonard (1974). In particular, suppose $H$ is an abelian normal subgroup of the (solvable) group $G$, with $G / H \simeq K$. Here we consider maps of the type $h \rightarrow h^{n}, h \in H$ and $\boldsymbol{n}$ an integer, as candidates for the permutations $\pi_{c}$.

THEOREM 4. Let $H$ be abelian, and suppose there is an adder for $P S(H)$, and a left-handed adder for PS $(K)$. In addition, suppose a positive integer $n$ exists such that 
(i) $(n,|H|)=1$,

(ii) $(n-1,|H|)=1$, and

(iii) $\left(n^{l}+1,|H|\right)=1$, where $l=\exp (K)$.

Then there is a left-handed adder for $\operatorname{PS}(G)$.

Proof. For any $c \in K$, let $\pi_{c}$ denote the map $h \rightarrow h^{n}$. Conditions (i) and (ii) guarantee that $\pi_{c}$ and $h \rightarrow\left(h \pi_{c}\right) h^{-1}$ are permutations of $H$. It is easy to see that the map $k \rightarrow\left(h \pi_{c}\right)^{c} h$ fails to be a permutation of $H$ if and only if there is an $h \neq 1$ in $H$ such that $h^{c^{-1}}=h^{-n}$. This implies $h=h^{\left(c^{-1)|c|}\right.}=h^{-n|c|}$, where $|c|$ denotes the order of the element $c$ in $K$. This implies $n^{|c|}+1 \equiv 0(\bmod |h|)$, which is not possible by (iii). The existence of a left-handed adder for $P S(G)$ now follows by Theorem 1 .

The wide applicability of Theorem 4 is now guaranteed by the following lemma.

Lemma. Let $N$ and $l$ be odd positive integers, with $3 \times N$. Then there is an integer $n, 0 \leqq n<N$, satisfying the three conditions

(i) $(n, N)=1$,

(ii) $(n-1, N)=1$,

(iii) $\left(n^{\prime}+1, N\right)=1$.

Proof. By the Chinese Remainder Theorem, it suffices to prove the lemma for $N=p^{k}$, where $p$ is a prime larger than 3 . As $p$ exceeds 3 , the set $S$ of integers $n, 0 \leqq n<p^{k}$ such that $(n, p)=(n-1, p)=(n+1, p)=1$ is nonempty. Let $n^{\prime} \in S$; then $n^{\prime \prime}=p^{k}-n^{\prime} \in S$. Moreover, either $n^{\prime}$ or $n^{\prime \prime}$ satisfies (iii), since $\left(n^{\prime}\right)^{l}+1 \equiv 0(\bmod p)$ implies $\left(n^{\prime \prime}\right)^{l}+1 \equiv 2(\bmod p)$. This completes the proof.

THEOREM 5. Suppose $H$ is abelian and $3 \nmid|H|$. Suppose that there exist an adder for and and a right-handed adder for PS(K). Then there exists a right-handed adder for PS $(G)$.

Proof. This follows from Theorem 4 and the above lemma.

Corollary 5.1. Let $H$ be abelian and $3 \nmid|H|$. Suppose that there exist an adder for PS $(H)$ and a right-handed adder for PS $(K)$. Then there exists a right-handed adder for PS $(G)$.

Proof. Let $\xi: G \rightarrow \bar{G}$ be an anti-isomorphism of $G$ onto $\bar{G}$. Then $H \xi=\bar{H}$ is abelian and normal in $\bar{G}$, and the map $\bar{\xi}: G / H \rightarrow \bar{G} / \bar{H}$ defined by $(g H) \bar{\xi}=$ $g \xi \bar{H}$ is an anti-isomorphism. Thus, $K$ is anti-isomorphic to $\bar{K} \simeq \bar{G} / \vec{H}$. Let $\eta: K \rightarrow \bar{K}$ be the anti-isomorphism. Since there is a right-handed adder $A$ for $P S(K)$ and $(P S(K)) \eta=P S(\bar{K})$, where $\eta$ is applied to $P S(K)$ in the natural manner, it follows that $\eta^{-1} A \eta$ is a left-handed adder for $P S(\bar{K})$. Now all of the conditions of Theorem 5 are satisfied by $\bar{H}, \bar{G}$ and $\bar{K}$, therefore there is a 
left-handed adder $B$ for $P S(\bar{G})$. But then $\xi B \xi^{-1}$ is a right-handed adder for $P S(G)$.

Using the above results, we are now in a position to show that there are adders for $P S(G)$ in "most" nonabelian groups $G$ of odd order. Before proceeding, we note that Theorem 5 has the following additional corollary which includes the results of Leonard (1974).

COROLlaRY 5.2. Let $G$ be any group of order $p q$, where $p$ and $q$ are distinct odd primes and $p, q \neq 3$ or 5. Then there exist both a left-handed and a right-handed adder for $P S(G)$.

Proof. Assume $p<q$. Let $H$ be the Sylow $q$-subgroup of $G$. $G$ is an extension of $H$, an abelian group, and by Gross and Leonard (to appear) there exist adders for $P S(H)$ and $P S(K)$; hence, by Theorem 5, there is a left-handed adder for $P S(G)$. By Corollary 5.1 there is a right-handed adder for $P S(G)$.

We now prove the result promised above.

THEOREM 6. Let $G$ be any group of order not divisible by 2,3 , or 5 . Then these exist both a left-handed adder and a right-handed adder for $P S(G)$.

Proof. We proceed by induction. Let $G$ be a group of minimal order such that the conditions of the theorem hold, but the conclusion fails to hold. Since $G$ is solvable, $G$ has a nontrivial normal abelian subgroup $H$. Also, by Gross and Leonard (to appear) we know that there is an adder for $P S(H)$, and by the induction hypothesis, we know that there exist both a left- and a right-handed adder for $P S(G / H)$. But by Theorem 5 and Corollary 5.1, this means that there must exist right- and left-handed adders for $P S(G)$, in contradiction to the hypothesis that one of these did not exist. This completes the proof.

It snould be pointed out that Theorem 5 in Gross and Leonard applies here also to show that if an abelian group $H$ has a cyclic Sylow 3-subgroup then there may be no workable possibility for the maps $\pi_{c}$ mentioned in Theorem 1 . Thus the condition $3 \nmid|H|$ is not superfluous in Theorem 5 above. However, the note contained in the proof of Corollary 4 of Gross and Leonard also applies here. In particular, the existence of what are known as skew strong starters, see Gross and Leonard, for many groups $\boldsymbol{H}$ of order divisible by 3 insures the existence of a permutation of $H$ such that $h \rightarrow(h \pi) \cdot h$ and $h \rightarrow(h \pi) \cdot h^{-1}$ are permutations of $H$. Such a permutation will satisfy the conditions of Theorem 1 if the map $h \rightarrow(h \pi)^{c} \cdot h$ is a permutation of $H$. In many simple cases $\pi$ is also an automorphism of $H$ : for example, this is the case with constructions of Mullin-Nemeth type (see Gross and Leonard Corollary 4, Gross (1974), and Mullin and Nemeth (1969)). In this case, letting $h \pi=h^{\pi}$ and replacing $n$ by $\pi$ in the proof of Theorem 4, we obtain the following sufficient condition for $\pi$ to be satisfactory (iii) $h \rightarrow h^{\pi^{\prime}} \cdot h$ is a permutation of $H$ (where $l=\exp (K)$ ). 
A major cause of failure of property (iii)' is the fact that $|\pi|||$ Aut $(H) \mid$, since we also have $l||$ Aut $(H) \mid$. One can avoid this by requiring that $(|\pi|, l)=1$. For example, there exists a Mullin-Nemeth construction yielding a skew strong starter for $H_{1}=Z_{3} \oplus Z_{3} \oplus Z_{3} \oplus Z_{3} \oplus Z_{3}$. From this starter one can obtain a permutation $\pi$ as described in Corollary 4 of Gross and Leonard. The order of $\pi$ is $3^{5}-1$ and $\pi$ is an automorphism of $H_{1}$; thus, condition (iii)' may fail to hold if $l \mid 3^{5}-1$. And, in fact $l=121$ does indeed fail to satisfy condition (iii)' for this $\pi$. It is permissible, however, to take $l=11=\left(3^{5}-1\right) / 22$ or to take $l=13=$ $\left(3^{3}-1\right) / 2$. Thus, we have shown the existence of left- and right-handed adders for $P S(G)$, where $G$ is any extension of $H_{1}$ by groups of orders 11 or 13 .

We leave it to the interested reader to study these extensions of the foregoing theorems in more detail.

\section{References}

B. A. Anderson (1973), 'Finite Topologies and Hamiltonian Paths', J. Combinatorial Theory Ser. B. 14, 87-93.

B. A. Anderson (1974), 'A Class of Starter Induced 1-Factorizations', Graphs and Combinatorics, Lecture Notes in Mathematics No. 406 (Springer-Verlag, Berlin-Heidelberg-New York, 1974).

K. Byleen (1970), ‘On Stanton and Mullin's construction of Room Squares', Ann. Math. Statist. 41, $1122-1125$.

W. Feit and J. G. Thompson (1963), 'Solvability of Groups of Odd Order', Pacific J. Math. 13, 775-1029.

K. B. Gross (1973), Constructions for inequivalent Room designs (Ph.D. Thesis, University of Southern California, 1973).

K. B. Gross (1974), 'Equivalence of Room designs II', J. Combinatorial Theory Ser. A 17, $299-316$.

K. B. Gross and P. A. Leonard (to appear), 'The Existence of Strong Starters in Cyclic Groups', Utilitas Math.

S. H. Y. Hung and N. S. Mendelsohn (1974), 'On Howell Designs', J. Combinatorial Theory Ser. A. 16, 174-198.

P. A. Leonard (1974), 'Adders for patterned starter in some non-abelian groups', Bull. Austral. Math.'Soc. 10, 321-323.

R. C. Mullin and E. Nemeth (1969a), 'An existence theorem for Room Squares', Canad. Math. Bull. 12, 493-497.

R. C. Mullin and E. Nemeth (1969b), 'On furnishing Room squares', J. Combinatorial Theory Ser. A 7, 266-272.

W. D. Wallis, A. P. Street and J. S. Wallis (1972), Combinatorics: Room Squares, Sum-Free Sets, Hadamard Matrices, Lecture Notes No. 292 (Springer-Verlag, Berlin-Heidelberg-New York, 1972).

W. D. Wallis, (1973), 'On one-factorizations of complete graphs', J. Austral. Math: Soc. 16, $167-171$.

Louisiana State University

Louisiana, 70803, U.S.A.

Arizona State University

Arizona, 85281, U.S.A. 